\title{
Indirect calorimetry in critical care
}

\author{
BY JAMES. L. MULLEN \\ Division of Metabolism and Nutrition, University of Pennsylvania School of Medicine, \\ Philadelphia, PA, USA
}

In critical care, particular attention is paid to disorders which lead to cerebral dysfunction. Hypoxia is the most common example and considerable effort is devoted to avoiding this problem. If the brain is deprived of fuel, as in hypoglycaemia, the outcome is similar yet far less attention is paid to maintaining an adequate and appropriate long-term fuel supply.

Indirect calorimetry has been investigated for the past decade, focusing on its application to the clinical area. The only pathway to the effective clinical use of indirect calorimetry is via a meticulous research-precision approach with considerable attention to accuracy. Our institution played a key role in the development of parenteral nutrition. In the early days, patients were provided $12 \cdot 6-20 \cdot 9 \mathrm{MJ}(3000-5000 \mathrm{kcal}) / \mathrm{d}$ with the 'thought' that 'hyper' alimentation was good. Energy requirements of sick patients were ill-defined and 'guess-work' at best. Energy prescription often consisted of 'three bottles/d' and such input often exceeded the capacity of the body's disposal systems. Unfortunately serious adverse events accompanied overfeeding: hyperglycaemia, hyperosmolar states, steatosis, excessive carbon dioxide production, increased norepinephrine secretion and fluid overload. When any or all these events occurred the temptation for the clinician was to abandon forced feeding and blame total parenteral nutrition (TPN). TPN is not to blame, only its misuse. We must approach energy prescription much as we approach medication prescriptions with precision based on scientific objective information.

\section{TECHNOLOGY}

Indirect calorimetry measures gas exchange in contrast to the heat exchange measurements of direct calorimetry. Multiple studies have validated their comparable results in varying settings. Indirect calorimeters can have a closed or an open circuit which is preferred by most. Although the mouthpiece or mask is still used occasionally, the canopy technique is preferred for clinical studies. A whole-room indirect calorimeter has research advantages but is impractical in patient care. Clinically useful indirect calorimeters must have portability, automated calibration, limited sensor drift, the ability to collect gas exchange under a variety of circumstances, flexibility in their computer software and a minimal warm-up time. A calibration checklist must be navigated, and the system must be checked for any leaks to maintain stable inspired oxygen concentration as well as maintenance of sufficient desiccant to remove excess humidity. Should the indirect calorimetry measurements be continuous over $24 \mathrm{~h}$ to measure a patient's total energy expenditure (TEE), or can a 'snapshot' measurement of resting energy expenditure (REE) be performed and extrapolated to an estimate of TEE? We measure a spot REE and extrapolate, controlling physical activity and nutrient intake; others have favoured random multiple 'snapshots' of non-resting measurements for more accurate extrapolation. REE test conditions include $0.5 \mathrm{~h}$ rest in bed, no 
nutrient intake within $2 \mathrm{~h}$ before the test and a thermoneutral environment. Gasexchange results must demonstrate a steady-state such that five consecutive $1 \mathrm{~min}$ measurements have a coefficient variation of less than $5 \%$. Using this rigid approach we can be confident that our 'snapshot' energy expenditure (EE) measurements are truly representative. If the measured REE is greater than $120 \%$ or less than $80 \%$ of expected, or if the minute ventilation is less than 5 litres or greater than 10 litres, or if the respiratory quotient (RQ) is greater than 1.00 we review the raw data to confirm an accurate collection. In 1980 , we showed a $2 \%$ coefficient of variation in REE measured multiple times during the same day (J. L. Mullen, unpublished results). The Rumpler et al. (1990) study shows that the coefficient of variation of REE measured on five consecutive days was $2 \%$ and only $3 \%$ when the REE was measured weekly for 1 month in the same subject. When TEE was measured for $24 \mathrm{~h}$, the daily measurements had a coefficient of variation of $3 \%$ and only $5 \%$ when done weekly (Rumpler et al. 1990). Bursztein et al. (1989) have clearly shown that the lack of nitrogen excretion values has little influence on EE calculations and can essentially be ignored. This is important clinically since coupling $\mathrm{N}$ excretion and $\mathrm{EE}$ measurements in sick patients is often difficult.

\section{MEASURING OR ESTIMATING EE?}

Whether one need measure EE in sick patients should no longer be debated. Multiple studies have adequately shown that the variability of measured $\mathrm{EE}$ compared with predictions for individuals is substantial; measured REE ranges from 50 to $150 \%$ of the expected metabolic rate in differing study populations (Feurer et al. 1984). Such wide variability compared with that expected precludes the use of a predictive formula to clinically define energy requirements, since the risk of substantial under- and overfeeding is unacceptably high. In our initial studies (Feurer et al. 1984), measured EE ranged from $57 \%$ to $154 \%$ of the Harris-Benedict prediction. The degree of variability is closely linked to disease severity and is due to the distorted body composition, abnormal metabolic activity per unit of tissue, and multiple thermogenic and thermo-depressive clinical interventions. For example, barbiturate coma suppresses EE and serum phenobarbital level is directly correlated with the metabolic rate (Dempsey et al. 1985). Head-trauma patients without phenobarbital therapy had a measured REE of $125 \%$ of the expected value and under barbiturate coma this was down to $60 \%$ of the expected value. If one used expected $\mathrm{EE}$ values for energy prescription, considerable under- and overfeeding would occur dependent on the serum barbiturate level. Small clinical procedures like biopsies and intubations have dramatic short-term effects on EE; however, their limited duration minimizes their overall contribution to TEE (Damask et al. 1983). Under normal starvation, the resultant reduction in EE is greater than that one would expect if it was totally due to the loss of metabolically active tissue. In addition to the reduction of metabolic activity from losing active tissue, there is a decrease in the metabolic activity per unit of remaining tissue. This has been repeatedly observed from Benedict's early studies through Keyes' Minnesota starvation experiments in the 1950s. Although we usually assume diseases uniformly elevate EE, in certain circumstances, such as a spinal cord injury, EE actually declines. In the Cox et al. (1985) study, quadriplegics had an EE $30 \%$ below the expected value and paraplegics were $10 \%$ less than the expected value. Sepsis, operations, trauma, respiratory failure all generate elevated EE in some but not all sick patients. The concept of attributing a certain degree 
of elevation of TEE to a given disease and extrapolating this to all patients with that disease regardless of severity and time-course is a serious error. In many patients there is often an initial hypermetabolism which slowly declines over time.

Of particular interest outside critical care, is the obese patient. No acceptable standards exist for predicting EE in obese patients because of their variably distorted body composition. If one assumes a normal body composition based on their current body-weight, their measured EE is considerably less than expected. If one assumes a normal body composition based on their ideal body-weight their measured EE is much higher than one would expect. Although we have tried repeatedly to develop an accurate predictive equation, it has not been possible with acceptably narrow $95 \%$ confidence limits.

Much to our surprise in our initial cancer study (Knox et al. 1983), we found certain cancer patients hypermetabolic compared with that expected, but also a substantial number who were hypometabolic; that is, their measured EE was less than what one would expect from a Harris-Benedict prediction. These metabolic aberrations obviously could be due to distorted body composition, effects of tumour metabolism directly, or effects of the tumour on host metabolism. Similar observations made us cautious in accepting the simple notion that a tumour's metabolic activity was a major contributor to cancer hypermetabolism and cachexia. Exploring this issue further, the anatomic site of the malignancy is a determinant of metabolic rate distortion. Hypermetabolism predominates in the gastric cancer, and lung cancer populations (Shike, 1981; Russell et al. 1984), whereas populations of pancreatic or hepatobiliary cancer patients are shifted towards hypometabolism (Dempsey et al. 1984). Our logical hypothesis that the degree of tumour burden would be correlated with metabolic rate has proved to be false. In colorectal cancer patients, hypermetabolism was no more common in patients with liver metastases compared with Dukes A localized disease patients. If tumour burden was influencing metabolism in a relatively simple way it should have been detected in this study. Hansell et al. (1986) proposed that aberrations in EE in cancer patients are due to abnormal body composition with no effect from the tumour. Our recent study provides interesting insight (Luketich et al. 1990). Patients undergoing curative resections had a normal post-operative EE regardless of whether their preoperative metabolic rate was elevated or depressed. We controlled for nutrient intake and all other influencing variables. Much to our surprise, patients who had a palliative resection had an abnormal post-operative EE. Metabolic rate was not normalized when the tumour was not removed and the trend was for such patients to become hypermetabolic regardless of their preoperative metabolic rate. Obviously the patient's body composition did not change appreciably in this short period so the dramatic changes in metabolic rate are attributable to removal or non-removal of the tumour.

\section{CLINICAL APPLICATIONS}

To clinically apply such information, we make assumptions and generalizations. Energy balance is synonymous with fat balance and if energy supply to the patient exceeds their $\mathrm{TEE}$, fat stores will increase. If the exogenous energy supply is less than TEE, body fat stores will decline over time. TEE consists of a resting component (REE), a component due to physical activity (activity $\mathrm{EE}$; $\mathrm{AEE}$ ), and a component due to dietary-induced thermogenesis (DIT), although this may only contribute when the rate of exogenous 
energy supply is higher than the metabolic rate. Measuring TEE for $24 \mathrm{~h}$ provides a precise measurement but is rather impractical. A number of investigators extrapolated TEE from REE measurements in hospitalized patients and have shown remarkable consistency with TEE in the range of $120 \%-140 \%$ of REE. Using that consensus, we calculated TEE to be $130 \%$ of measured REE for hospitalized patients. This calculation is necessarily different if the AEE component of TEE is substantially higher, for example, in free-living outpatients. Hospitalized patients have limited physical activity but only for a short period and the overall contribution to TEE is less than one might expect.

Having determined TEE, our energy prescription is based on our goal for the patient's fat stores. If the patient's current weight is less than $90 \%$ of ideal weight, their fat stores are subnormal and we prescribe an energy supply to replete those fat stores (energy supply $>$ TEE). How much greater depends on how rapidly we want to restore fat stores and the ability of the patient's liver to convert and export the energy overload to peripheral fat stores. If the patient's current weight is between 90 and $120 \%$ of their ideal body-weight, their fat stores are normal and our energy goal is to maintain those fat stores at their current size (energy supply = TEE). If their dry current body-weight is greater than $120 \%$ of their ideal body-weight, their fat stores are excessive and we would not be unhappy if they were depleted over the hospital course. We supply energy at a rate less than their TEE, driving the cannibalization of their endogenous fat stores. This approach has worked well and has brought rationality to the energy prescription process.

If a patient is 'overfed' with energy, the excess is stored as fat. This occurs mainly in the liver, and hepatic steatosis and 'dysfunction' are not an uncommon complication of TPN when excessive energy content is used. In our institution, we have rarely seen abnormal liver function tests after we started providing energy based on the actual measurement of EE. This is an important advance because the development of 'hepatic dysfunction' often leads the non-nutrition clinician to conclude that forced-feeding is bad and they stop feeding the patient. The problem is the poor prescription and not the technology.

It has been speculated that glucose and fat as energy sources are clinically different in terms of increased $\mathrm{CO}_{2}$ production. If energy is supplied at a rate below TEE, $\mathrm{CO}_{2}$ production is only elevated $8 \%$ above what is generated when the patient is starving without exogenous energy (Compher \& Mullen, 1990). During such starvation the patient's endogenous fat stores are burned, generating $\mathrm{CO}_{2}$. Only when exogenous energy is supplied at a rate above TEE is $\mathrm{CO}_{2}$ production increased substantially, with the generation of obvious problems if the respiratory disposal system is inadequate. When energy supply is at or below TEE, there is little difference in $\mathrm{CO}_{2}$ production between a totally glucose and a glucose-fat $(50: 50$, w/w) regimen. These findings question whether there is any great benefit in altering energy source to manipulate $\mathrm{CO}_{2}$ production when supplying energy at or below TEE.

We have explored the hypothesis that one may simultaneously create a positive clinical outcome, positive $\mathrm{N}$ balance and negative energy or fat balance. Our study population was morbidly obese patients with over $1674 \mathrm{MJ}$ (400 $000 \mathrm{kcal})$ of fat stores who had an intra-abdominal abscess and enterocutaneous fistula and were 'sick' with these potentially lethal post-operative complications (Dickerson et al. 1986). It made no sense providing sufficient energy to maintain or expand the fat stores in patients who already had an excessive amount, so energy supply was at only $30 \%$ of their TEE. By design the 
other energy source would be their endogenous fat stores so these fat stores and body-weight would gradually decline. We provided protein at the rate of $2 \mathrm{~g} / \mathrm{kg}$ ideal body-weight. The patients lost $1.5 \mathrm{~kg}$ weight/week primarily as fat, as evidenced by their $\mathrm{RQ}$ of approximately $\mathbf{0 . 7 5}$. For the clinician, the results were dramatic. All fistulas healed and they were in $\mathrm{N}$ balance or slightly positive at $+2 \mathrm{~g} / \mathrm{d}$ and their serum proteins gradually returned to normal. One can achieve positive $\mathrm{N}$ balance, get wound healing and closure of enterocutaneous fistulas despite a negative energy balance.

Foster et al. (1987) reviewed the guidelines for energy requirements published in the literature. They used 100 malnourished TPN patients who were fed for more than $7 \mathrm{~d}$. Their measured EE had the usual variability in that only $48 \%$ had a measured EE within $90-110 \%$ of the expected rate. We compared energy prescription based on their actual measured TEE with the energy prescription based on all available applicable recommendations in the literature. The published guidelines recommended supplying from $2 \cdot 1$ to $8.4 \mathrm{MJ}$ ( 500 to $2000 \mathrm{kcal}$ ) per d more than the recommendation based on actual measured TEE. Blindly following such published guidelines would lead to considerable overfeeding and all its adverse clinical events as well as economic disadvantages. In our institution in 1 year, using a measured TEE-based energy prescription methodology, we reduced the total volume of TPN from 33000 litres/year to 26000 litres/year; this reduction in TPN of over 7000 litres/year is not inconsequential.

Our overall philosophy for energy prescription in the critically ill patient emphasizes a balanced approach with the provision of energy at the lowest level possible to achieve maximal benefit. We must be cognizant of the limitations of the respiratory, renal or hepatic disposal systems. There is no obvious benefit in creating or maintaining obesity and no indication as to why we should 'fatten-up' the patient. Endogenous fat can provide for energy needs and with this rational approach to energy prescription there is a great reduction in the number of complications of excessive energy delivery.

One must always be conscious that the efficacy of one's clinical service might be limited by how well one can deliver the energy prescribed. In a recent review of an intensive care unit, we found that the 'system' was only able to deliver $70 \%$ of the energy prescribed (Evans et al. 1991). Parenteral $v$. enteral route was not a factor. The main reasons for non-delivery were logistical problems, e.g. no available access, fluid restrictions or decreased clearance through disposal systems. If one can only deliver $70 \%$ of the prescribed energy, it makes little sense to expend considerable resources to precisely define unachievable energy goals.

\section{REFERENCES}

Bursztein, S., Saphar, P., Singer, P. \& Elwyn, D. H. (1989). A mathematical analysis of indirect calorimetry measurements in acutely ill patients. American Journal of Clinical Nutrition 50, 227-230.

Compher, C. W. \& Mullen, J. L. (1990). Parenteral caloric supply and $\mathrm{CO}_{2}$ production. Clinical Nutrition 9 , 29.

Cox, A. R., Weiss, S., Posuniak, R. A., Worthington, P., Prioleau, M. \& Heffley, G. (1985). Energy expenditure after spinal cord injury: an evaluation of stable rehabilitating patients. Journal of Trauma $\mathbf{2 5}$, $419-423$.

Damask, M. C., Askanazi, J., Weissman, C., Elwyn, D. H. \& Kinney, J. M. (1983). Artifacts in measurement of resting energy expenditure. Critical Care in Medicine 11, 750-753.

Dempsey, D. T., Feurer, I. D., Crosby, L. O., Knox, L. S., Buzby, G. P. \& Mullen, J. L. (1984). Energy expenditure in malnourished gastrointestinal cancer patients. Cancer 53, 1265-1273. 
Dempsey, D. T., Guenter, P., Mullen, J. L., Fairman, R., Crosby, L. O., Spielman, G. \& Gennarelli, T. (1985). Energy expenditure in acute head trauma with and without barbiturate therapy. Surgery in Gynecology and Obstetrics 170, 128-134.

Dickerson, R. N., Rosato, E. F. \& Mullen, J. L. (1986). Net protein anabolism with hypocaloric parenteral nutrition in obese stressed patients. American Journal of Clinical Nutrition 44, 747-755.

Evans, N. J., Sorouri, B. B. \& Feurer, I. D. (1991). Constraints of nutrient supply in the intensive care unit. Journal of Parenteral and Enteral Nutrition 15, $34 \mathrm{~S}$.

Feurer, I, D., Crosby, L. O. \& Mullen, J. L. (1984). Measured and predicted resting energy expenditure in clinically stable patients. Clinical Nutrition 3, 27-34.

Foster, G. D., Knox, L. S., Dempsey, D. T. \& Mullen, J. L. (1987). Caloric requirements in total parenteral nutrition. Joumal of American College of Nutrition 6, 231-253.

Hansell, D. T., Davies, I. W. \& Burns, H. J. (1986). The effects of resting energy expenditure of different tumor types. Cancer 58, 1739-1744.

Knox, L. S., Crosby, L. O., Feurer, I. D., Buzby, G. P., Miller, C. L. \& Mullen, J. L. (1983). Energy expenditure in malnourished cancer patients. Annals of Surgery 197, 30-40.

Luketich, J. D., Mullen, J. L., Feurer, I. D., Sternlieb, J. \& Fried, R. C. (1990). Ablation of abnormal energy expenditure by curative tumor resection. Archives of Surgery 125, 337-341.

Rumpler, W. V., Seale, J. L., Conway, J. M. \& Moe, P. W. (1990). Repeatability of 24-hr energy expenditure measurements in humans by indirect calorimetry. American Journal of Clinical Nutrition 51, 147-152.

Russell, D. M., Shike, M. \& Marliss, E. B. (1984). Effects of TPN and chemotherapy on the metabolic derangements in small cell lung cancer. Cancer Research 44, 1706-1711.

Shike, M. (1981). Energy expenditure in relation to caloric intake. Journal of Parenteral and Enteral Nutrition 5,562 . 